\title{
La mesure de la vitesse de la lumière à Nice par Henri Joseph Anastase Perrotin
}

Gilles Bogaert ${ }^{(1)}$ (bogaert@oca.eu) et Wilfried Blanc ${ }^{(2)}$ (wilfried.blanc@unice.fr) (1) ARTEMIS, UNS-0CA-CNRS, 06304 Nice Cedex

(2) Laboratoire de Physique de la Matière Condensée, CNRS-UNS, 06108 Nice Cedex

Entre 1898 et 1904, Perrotin,

directeur du nouvel

Observatoire de Nice [1], entreprend de mesurer la

vitesse de la lumière avec une précision record, qui entrera dans I'histoire. Cette entreprise, quasiment oubliée aujourd'hui, a pourtant donné un résultat cité comme référence pendant des décennies.

Les archives de l'Observatoire de

la Côte d'Azur [2] (encadré p. 23) permettent de revivre au jour le jour ce projet, assez audacieux, et de redécouvrir cet homme déterminé qui avait accepté de présider à la destinée de ce " palais astronomique et physique » pour la gloire de la « Science française »[3].
Après les mesures pionnières de Fizeau en 1849 et Foucault en 1862 (voir tableau p. 22), c'est encore un Français, Alfred Cornu, qui s'illustre en 1874 en mesurant la vitesse de la lumière avec précision : $300400 \pm 300 \mathrm{~km} / \mathrm{s}$. Il utilise pour cela un dispositif de roue dentée, dérivé de celui de Fizeau, décrit dans un article exceptionnel de plus de 300 pages. Cette « œuvre forte et durable » [4] lui vaut, entre autres, le prix Lacaze de l'Académie des sciences. Toutefois, de 1878 à 1883, Albert Michelson puis Simon Newcomb, par la méthode du miroir tournant, prétendent obtenir des précisions de 50 puis $30 \mathrm{~km} / \mathrm{s}$ ! Les valeurs qu'ils obtiennent ( $299860 \mathrm{~km} / \mathrm{s})$ sont strictement incompatibles avec celle de Cornu.

C'est dans ce cadre historique de controverse que Perrotin (fig. 1) décide, en 1897, de reprendre le flambeau en améliorant cette mesure "de nature à mettre en relief l'Observatoire » de Nice [5]. Il dispose pour cela de quelques atouts : pour améliorer la précision, il faut augmenter le nombre de mesures - le climat de Nice y est favorable et améliorer leur précision individuelle en utilisant des distances accrues, ce que les optiques puissantes de l'Observatoire devraient rendre possible. Il bénéficie aussi des soutiens indispensables de Cornu, qui confie son système de mesure (fig. 2), et de l'Observatoire de Paris qui fournit ses instruments de précision pour la mesure des distances. Il a aussi, bien sûr, le soutien indéfectible de Bischoffsheim, mécène et fondateur de l'Observatoire de Nice.

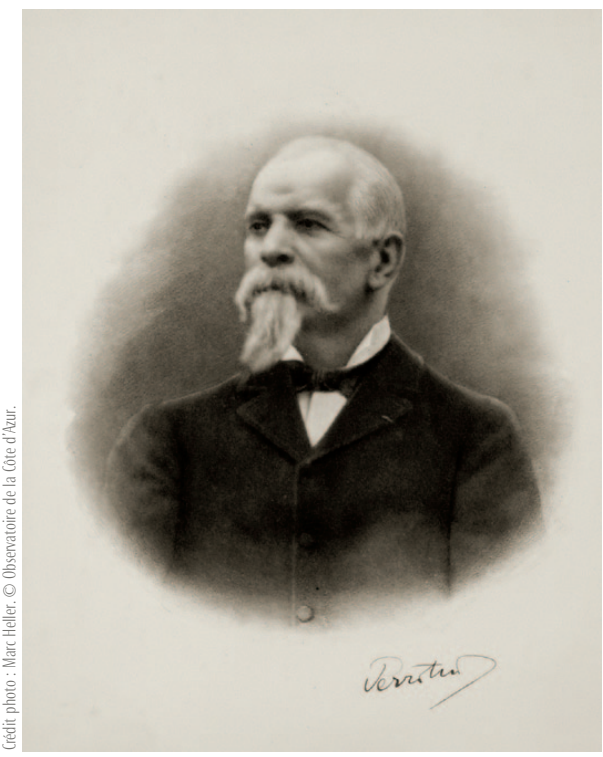

1. Portrait de Perrotin (1845-1904).

\section{Mesures préliminaires,} en préparant celle avec la Corse

Les carnets d'expériences (fig. 3) de Perrotin et des deux astronomes, Prim et Javelle, commencent le 18 février 1898, par des modes d'emploi : l'appareillage de Cornu est complexe et demande une prise en main. Par exemple, l'enregistreur consiste en un cylindre d'un mètre de circonférence, entouré d'un papier blanc qu'il faut noircir à la fumée, sur lequel viennent gratter quatre aiguilles mues par des électro-aimants reliés par des contacts électriques à l'horloge, à un compte-tour et au manipulateur Morse. Tout en actionnant le frein pour moduler la vitesse de la roue entraînée par un poids, il faut toper le contact Morse lors des apparitions et disparitions du faisceau de lumière, qui revient entre les minuscules dents de la roue. Cornu a d'ailleurs pris le soin d'être présent lors du démarrage. 


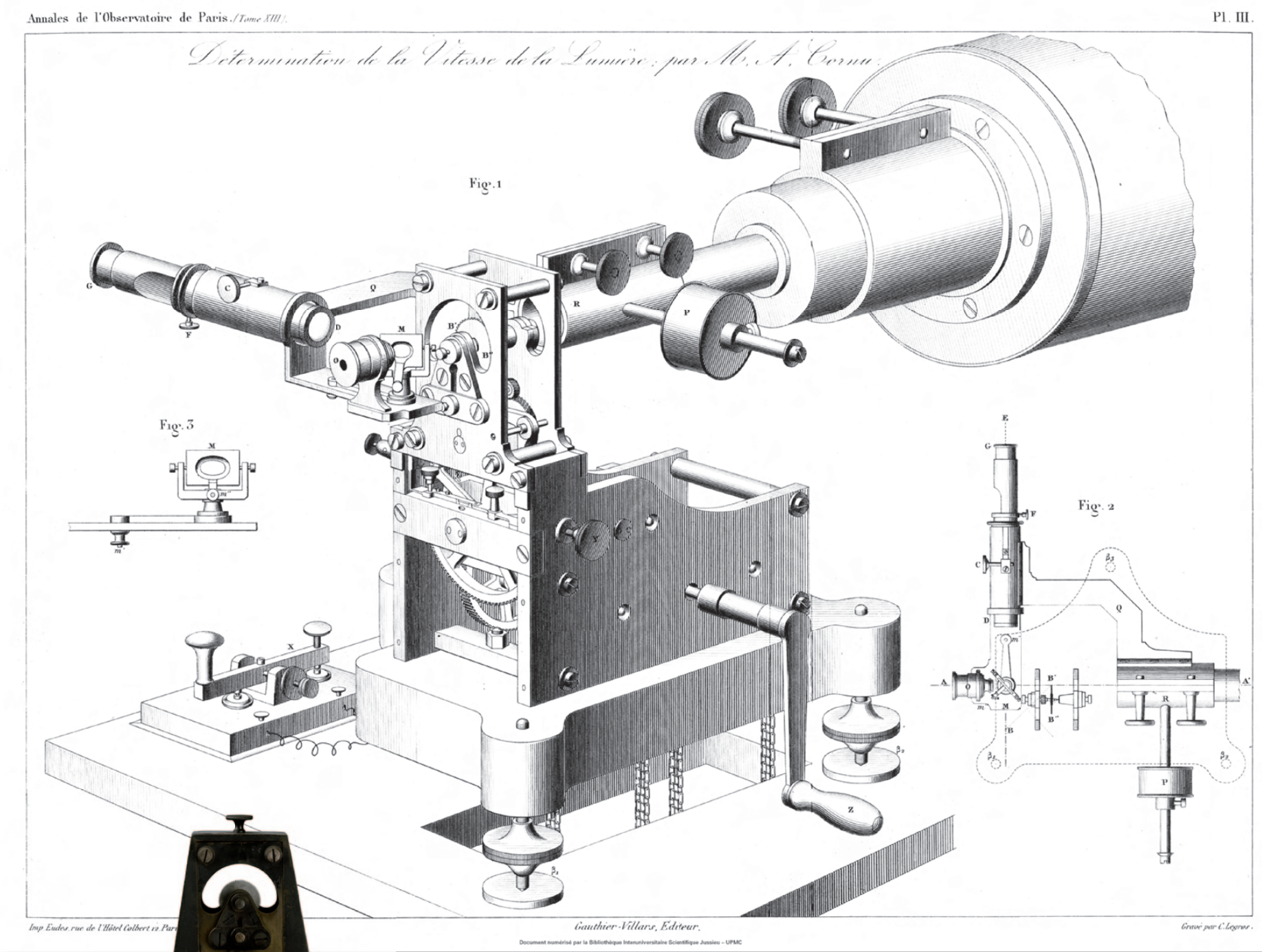

2. Appareil ayant servi à Cornu et Perrotin pour mesurer c. (a) Schéma du dispositif de mesure. La lumière de la lampe (non représentée) passe la roue dentée et entre dans le télescope à droite. La manivelle sert à remonter les poids qui, en tombant, font tourner la roue dentée à travers un engrenage. (Planche issue de la publication d’A. Cornu, Annales de l'Observatoire de Paris : Mémoires, Tome XIII (1876).) (b) Photo de l'appareil. On aperçoit la roue dentée (disque de couleur grise en haut).

mesure de Cornu reposait sur une distance de $22,91 \mathrm{~km}$; lui vise $250 \mathrm{~km}$ entre le Mont Mounier (à l'altitude de $2727 \mathrm{~m}$ ), qui a été acquis par Bischoffheim pour l'Observatoire, et un des hauts sommets corses! En septembre 1898, il se rend en Corse. Après avoir gravi les monts Rotondo et Cinto (2706 m), c'est ce dernier qu'il choisit pour accueillir, dans le futur, le collimateur de renvoi. De retour à Nice, les mesures vers La Gaude reprennent avec soin jusqu'en novembre 1898. Pour l'étape suivante, c'est le Mont Vinaigre, le plus haut sommet de l'Estérel, qui a été sélectionné, à $46 \mathrm{~km}$ vers l'Ouest. La distance va être mesurée à quelques centimètres près par Simonin, spécialiste de l'Observatoire. Prim y a fait une reconnaissance et fait construire un pilier pour recevoir le collimateur et son miroir. Malgré le soin apporté aux réglages, la lumière de retour ne sera que rarement visible. Perrotin comprend

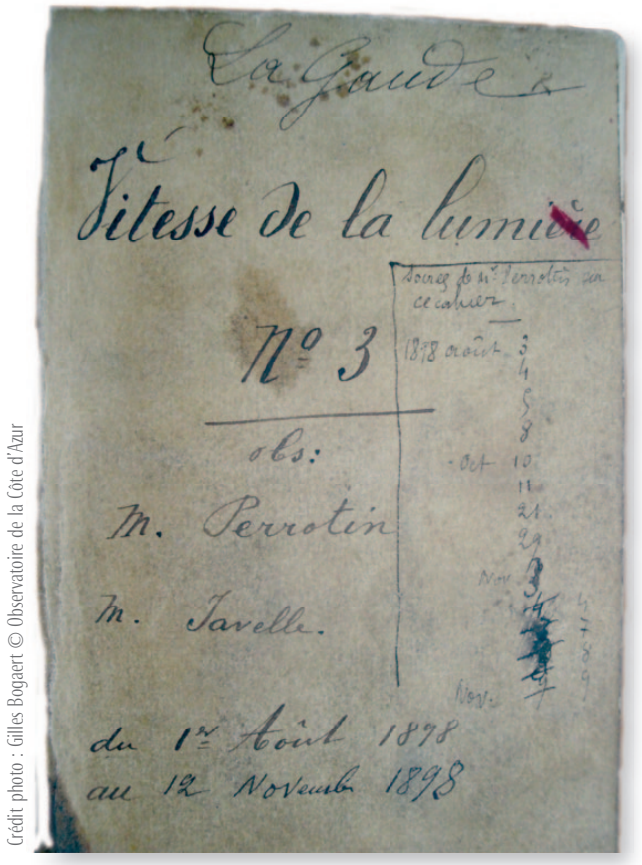

3. Couverture du Carnet 3 de Perrotin et Javelle. faire mieux que ses prédécesseurs, il envisage une mesure sur une distance extrême. La 
que l'air est très turbulent le soir le long du trajet, et le collimateur est trop étroit pour capter assez de lumière. Le temps d'améliorer le dispositif expérimental, de mobiliser les deux plus grandes lentilles de l'Observatoire, le temps aussi d'effectuer une remise en condition des appareils, les mesures ne reprendront que fin 1901.

\section{Le désaccord avec Cornu}

Entre-temps, en août 1900, a eu lieu à Paris le Premier Congrès International de Physique, présidé par Cornu. Dans sa revue des mesures de la vitesse de la lumière [6], il développe l'idée que le désaccord entre les résultats, le sien et ceux des Américains, n'est pas le fait des hommes, tous excellents, mais celui des méthodes, plus exactement des défauts de la méthode du miroir tournant, le principal étant que la vitesse latérale de l'image de la source, proche de celle de la lumière, ne peut être négligée [7]. Cornu argumente que la seule méthode fiable, car ne posant aucun problème théorique, est celle de la roue dentée, avec laquelle on obtient une valeur forcément supérieure à $300100 \mathrm{~km} / \mathrm{s}$. Bien qu'il connaisse parfaitement les résultats de Perrotin, il n'en fait pas mention [8].

Ce n'est qu'en novembre 1900, soit deux ans après la fin des mesures, que le résultat de La Gaude finit par être divulgué à l'Académie des sciences [5] : $299900 \pm 80 \mathrm{~km} / \mathrm{s}$, ramené au vide [9], donc en dehors de la fourchette de valeurs défendues par Cornu... Perrotin écrit de façon sibylline que ce résultat est " voisin de celui auquel a été conduit, dans ces dernières années, M. Michelson, par la méthode du miroir tournant de Foucault ». Ce résultat provoque des remous : le rapport annuel de l'Observatoire de Nice, adressé en 1901 par Perrotin au conseil de l'Université, va jusqu'à mentionner que «la publication, dans les CR de novembre dernier, des résultats fournis par la station de La Gaude (12 km), sujets, semble-t-il à quelques critiques, rend encore plus urgentes les déterminations que l'on propose d'entreprendre [avec le Mont Vinaigre]".

C'est en 1902 que se déroulent entre Nice et le Mont Vinaigre les mesures qui doivent améliorer la précision et mobilisent à cette fin les deux lunettes les plus puissantes de l'Observatoire : des milliers de mesures méticuleuses ont lieu tout au long de l'année, les soirs de grand calme dans le bâtiment du Grand Équatorial. La distance a été mesurée avec grand soin. C'est aussi en avril 1902 que Cornu décède... Aux funérailles de Cornu, Poincaré affirme, au sujet de la vitesse de la lumière : "Il est certain maintenant que le chiffre définitif ne pourra pas s'écarter beaucoup de celui qu'il [Cornu] a trouvé ", soit $300400 \mathrm{~km} / \mathrm{s}$.. . Mais en novembre 1902, jugeant qu'il a assez de données, Perrotin annonce son nouveau résultat à l'Académie des sciences, $299880 \pm 50 \mathrm{~km} / \mathrm{s}$ [10]. La précision obtenue est comparable à celle de Michelson et Newcomb... mais il confirme encore une fois leurs résultats, et non celui de Cornu!

\section{Une énigme épineuse, un résultat remarquable}

Préparant son expérience extrême, Perrotin a fait construire au Mont Mounier une cabane destinée à accueillir le dispositif de mesure. La visibilité de la Corse depuis le Mont Mounier a aussi fait l'objet d'études systématiques, tous les jours depuis janvier 1897. En 1903, fort de son expérience avec le Mont Vinaigre, Perrotin demande le financement d'un collimateur de grande dimension, aussi grand que la lunette du Grand Équatorial, pour optimiser le renvoi de la lumière après le trajet de $250 \mathrm{~km}$ dans l'air. Enfin et surtout, Perrotin a compris que des erreurs systématiques, qu'il a cherché à minimiser, ne s'éliminent pas totalement, contrairement aux allégations de Cornu. Il le mentionne au Comité

Valeurs de la vitesse de la lumière mesurées depuis 1849.

\begin{tabular}{|c|c|c|c|}
\hline Auteur année & Résultat (km/s) & Incertitude $(\mathrm{km} / \mathrm{s})$ & Remarques \\
\hline Fizeau 1849 & 304000 & & Roue dentée. Pas d'incertitude. \\
\hline Foucault 1862 & 298000 & 500 & Miroir tournant. Incertitude contestée. \\
\hline Cornu 1871 & 298500 & 1000 & Roue dentée. Mesure disqualifiée par Cornu en 1874. \\
\hline Cornu 1874 & 300400 & 300 & Première valeur non contestée. \\
\hline Michelson 1879 & 299910 & 50 & Miroir tournant. \\
\hline Young et Forbes 1881 & 301382 & & Mesure contestée dès la publication. \\
\hline Newcomb 1882 & 299860 & 30 & Miroir tournant. \\
\hline Michelson 1883 & 299853 & 60 & Miroir tournant. \\
\hline Abraham 1900 & 300010 & & Méthode non optique. \\
\hline Perrotin 1900 & 299900 & 80 & Roue dentée. \\
\hline Perrotin 1902 & 299880 & 50 & Le résultat final est publié en 1908 par Prim. \\
\hline Rosa et Dorsey 1907 & 299790 & 30 & Méthode non optique. \\
\hline Mercier 1922 & 299790 & 20 & Méthode non optique. \\
\hline Michelson 1927 & 299796 & 4 & Miroir modifié. \\
\hline c actuel 1983 & 299792,458 & 0 & Valeur définitivement fixée en 1983. \\
\hline
\end{tabular}




\section{L'Observatoire de la Côte d'Azur (OCA) aujourd'hui}

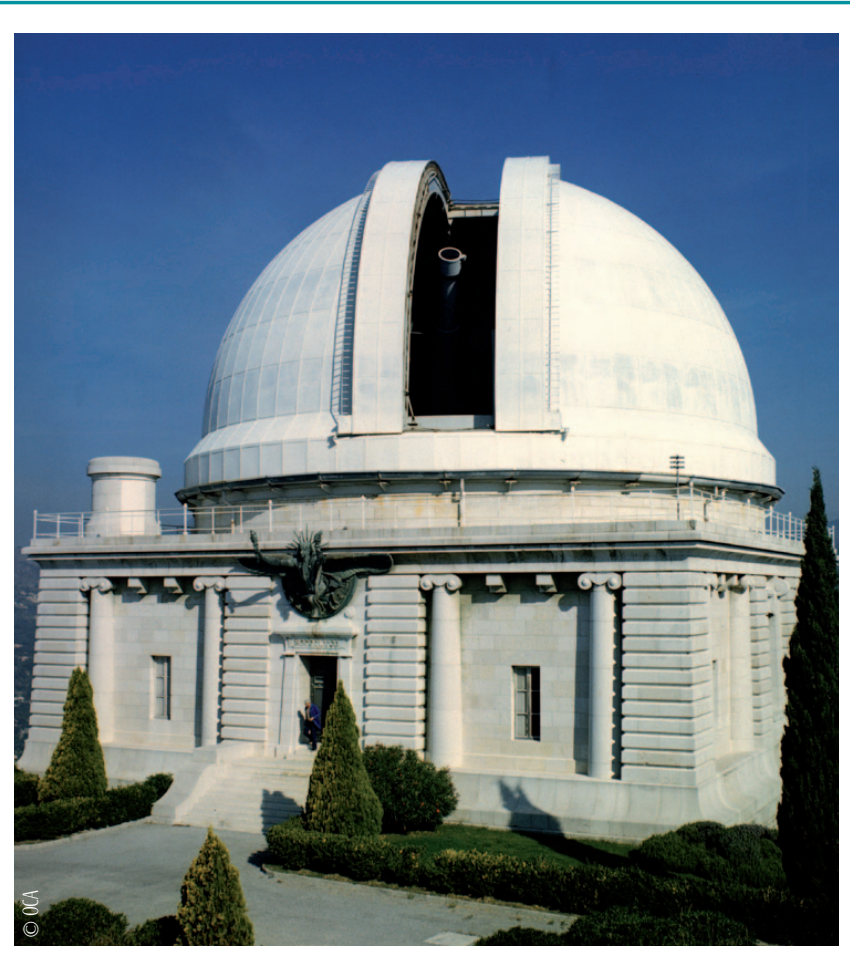

Les recherches menées à l'Observatoire de la Côte d'Azur concernent les sciences de la Terre et de l'Univers : la dynamique de la lithosphère, avec des enjeux importants sur les aléas sismiques et la surveillance du niveau des mers, et quasiment tous les domaines de l'astronomie et de la cosmologie. Les quatre UMR de l'Observatoire sont notées $\mathrm{A}^{+}$par I'AERES et sont chacune dans leur domaine des leaders internationaux qui attirent des étudiants et chercheurs du monde entier. Elles interviennent dans des grands projets comme GAIA, VIRGO, le VLTI, Concordia, avec une large gamme de savoir-faire et expertise, en instrumentation, dans le domaine des lasers, de l'imagerie, du calcul...

L'OCA possède aussi un patrimoine architectural et instrumental exceptionnel, au Mont Gros à Nice, œuvre de Garnier, et sur le plateau de Calern au-dessus de Grasse. II est implanté en tout sur sept sites, dont celui de Rustrel, dans le Var, et le parc de Sophia-Antipolis. Il développe des outils et projets pour la communauté éducative, comme Sismos à I'École et le Centre Pédagogique Planète Univers (C2PU), utilisant deux télescopes d'un mètre de diamètre pour de nouvelles méthodes pédagogiques en Licences et Masters.

Photo du Grand Équatorial, situé sur le Mont Gros à Nice. Architecte Charles Garnier, statue de bronze de Bayard de la Vingterie "Apollon sortant du Zodiaque ", et coupole de Gustave Eiffel. On aperçoit la grande lunette, longue de $18 \mathrm{~m}$, munie d'un objectif de $76 \mathrm{~cm}$ de diamètre.

de Direction, où siègent Poincaré et Lippmann [11]. Il a installé un banc de test dans le Grand Équatorial et une mire sur le Mont Macaron à $6 \mathrm{~km}$ au Nord, pour améliorer le dispositif. Mais Perrotin décède le 29 février 1904, brusquement. Personne n'est en mesure de continuer ses travaux. En 1908, les Annales de l'Observatoire de Nice publieront l'article commencé par Perrotin et fini par Prim, après relecture par le Comité de Direction. Les résultats de Perrotin figureront au début du $20^{\mathrm{e}}$ siècle dans les tables de constantes physiques.

L'histoire des sciences n'a pas fait grand cas de Perrotin et de cette mesure de c. On imagine que le désaccord entre ses résultats et celui de Cornu jeta la consternation, en constituant une énigme épineuse : le dispositif utilisé était le même, la méthode due à Cornu, celui-ci étant réputé suivre les travaux de Perrotin ! Aujourd'hui, la vitesse de la lumière (299 792, $458 \mathrm{~km} / \mathrm{s})$, est connue et on sait donc que la mesure de Cornu, en 1874 était erronée, malgré ses précautions et sa minutie extrêmes, pour une ou des causes à jamais inconnues. Leur origine est peut- être, comme le dit Perrotin, à chercher dans les erreurs systématiques qu'il a découvertes. Mais quelle qu'en soit la raison, il est remarquable que Perrotin ait réussi à obtenir un résultat plus exact, avec le même dispositif. C'est sans doute le fruit de son exigeante rigueur, et de son expérience des mesures de précision en astronomie. Mais, pour son malheur, la valeur obtenue, en désaccord avec celle de

\section{Références}

1- Perrotin est directeur depuis 1801 de l'0bservatoire de Nice, inauguré en 1887. Voir les Annales de l'Observatoire de Nice, vol. 1 (1899).

2• Observatoire de la Côte d'Azur, Av. de l'Observatoire. 06304 Nice

3• Annales de l'Observatoire de Nice, vol. 1 (1899).

4 Selon son rapporteur, H. Fizeau. Le Verrier fera aussi des louanges sur le traitement des erreurs.

5• Comptes rendus hebdomadaires des séances de l'Académie des sciences, Tome XXXXI, p. 731, séance du lundi 5 novembre 1900, « Sur la vitesse de la lumière », note de M. Perrotin présentée par M. Cornu.

$6 \bullet$ "Sur la vitesse de la lumière ", A. Cornu, Congrès International de Physique, Paris, Gauthier-Villars (1900).
Cornu, n'était pas facilement acceptable en France. Au lieu des honneurs légitimes, il reçut des critiques. Si Perrotin avait pu continuer, la mesure vers la Corse, par son aspect spectaculaire propre à frapper l'imagination, serait certainement entrée dans l'histoire. Et sa détermination lui aurait peut-être permis d'atteindre une précision de quelques km/s, comme Michelson en 1927 , après de nombreuses années de travail.
7- Cet argument, on le sait aujourd'hui, ne tient pas, car la vitesse latérale d'une image n'a rien à voir avec la vitesse des photons. D'ailleurs, une tache de lumière peut se déplacer à des vitesses bien supérieures à celle de la lumière.

8 Une note en bas de page mentionne, en tout et pour tout, que « des expériences en cours d'exécution à l'Observatoire de Nice, ont porté la distance à 40 km, c'est-à-dire avec un trajet de 80 km pour la lumière. "

9• La correction due à l'air est de $80 \mathrm{~km} / \mathrm{s}$.

10• H. Perrotin, « Vitesse de la lumière ; parallaxe solaire », CRAS (24 nov 1902), Tome CXXXV, p. 881

11• Lippmann recevra le Prix Nobel en 1908 pour ses travaux sur la photographie couleur.

D'autres documents sont accessibles sur le site http://canice.unice.fr 